\title{
Testing Kerr black hole mimickers with quasi-periodic oscillations from GRO J1655-40
}

\author{
Xin Jiang ${ }^{1, \mathrm{a}} \mathbb{C}_{\mathbb{C}}$, Peng Wang ${ }^{1, \mathrm{~b}}$, Houwen $\mathrm{Wu}^{1,2, \mathrm{c}}$, Haitang Yang ${ }^{1, \mathrm{~d}}$ \\ ${ }^{1}$ Center for Theoretical Physics, College of Physics, Sichuan University, Chengdu 610064, China \\ 2 Department of Applied Mathematics and Theoretical Physics, University of Cambridge, Wilberforce Road, Cambridge CB3 0WA, UK
}

Received: 4 August 2021 / Accepted: 6 November 2021

(C) The Author(s) 2021, corrected publication 2022

\begin{abstract}
The measurements of quasi-periodic oscillations (QPOs) provide a quite powerful tool to test the nature of astrophysical black hole candidates in the strong gravitational field regime. In this paper, we use QPOs within the relativistic precession model to test a recently proposed family of rotating black hole mimickers, which reduce to the Kerr metric in a limiting case, and can represent traversable wormholes or regular black holes with one or two horizons, depending on the values of the parameters. In particular, assuming that the compact object of GRO J1655-40 is described by a rotating black hole mimicker, we perform a $\chi$-square analysis to fit the parameters of the mimicker with two sets of observed QPO frequencies from GRO J1655-40. Our results indicate that although the metric around the compact object of GRO J1655-40 is consistent with the Kerr metric, a regular black hole with one horizon is favored by the observation data of GRO J1655-40.
\end{abstract}

\section{Contents}

1 Introduction . . . . . . . . . . . . 1

2 Epicyclic frequencies . . . . . . . . . . . . . 2

3 Rotating Simpson-Visser metric . . . . . . . . . . 3

4 Constraining rotating Simpson-Visser metric by quasi-periodic oscillations . . . . . . . . . 5 5

5 Conclusions ............... 6

References . . . . . . . . . . . . . . . 7

\footnotetext{
a e-mail: xjang@stu.scu.edu.cn (corresponding author)

be-mail: pengw@scu.edu.cn

c e-mail: hw598@damtp.cam.ac.uk

de-mail: hyanga@scu.edu.cn
}

\section{Introduction}

The first observations of gravitational waves by LIGO [1] and the first image of a black hole in the galaxy M87 [2] have ushered us into a new era of testing general relativity (GR) in the strong gravity regime. On the other hand, quasi-periodic oscillations (QPOs) are observed in the X-ray flux from black hole and neutron star X-ray binary systems, and detected as narrow peaks in the power density spectrum [3]. QPOs are believed to be associated with motion and accretionrelated timescales in a region of order the Schwarzschild radius around the compact object, which makes QPOs excellent probes of the strong gravitational field regime [4-8]. In black hole systems, the observed frequencies of QPOs range from $\mathrm{mHz}$ to hundreds of $\mathrm{Hz}$. While low frequency QPOs $(\lesssim 30 \mathrm{~Hz})$ are commonly observed from black hole X-ray binaries [9], high frequency QPOs $(\gtrsim 60 \mathrm{~Hz})$ are very rare. In fact, the Rossi X-ray Timing Explorer (RXTE), operational between 1996 and 2012, first detected high frequency QPOs in black hole systems [10,11]. Interestingly, a pair of simultaneous high-frequency QPOs was first discovered in the X-ray flux from GRO J1655-40 by RXTE [12]. It was noted that the frequencies of the two high-frequency QPOs are in a 3:2 ratio, suggesting a resonance between orbital and epicyclic motion of accreting matter near the innermost stable circular orbit (ISCO) of black holes [13]. Later, three simultaneous QPO frequencies, consisting of two higher frequencies and one lower frequency, were also observed from the X-ray data of GRO J1655-40 [14].

On the theoretical side, various models have been proposed to explain QPOs by relating them to the orbital and epicyclic frequencies of geodesics, such as the relativistic precession model (RPM) [15], the tidal disruption model [16], the parametric resonance model [17], the resonance model $[13,18,19]$, the warped disk oscillation model [20] and the non-axisymmetric disk oscillation model [21,22]. With these models, the observation data of QPO frequencies 
have been used to constrain the parameters of X-ray binary systems and test the nature of various gravity theories [2337].

Particularly, it showed that the X-ray data of GRO J165540 , especially the QPO triplet, fit nicely in the RPM, and the mass and spin of the compact object of GRO J1655-40 can be precisely determined [14]. Remarkably, the inferred mass is in great agreement with the dynamical mass measurement [23]. The RPM was originally proposed to explain QPOs in low-mass X-ray binaries with a neutron star [38], and later extended to systems with stellar-mass $\mathrm{BH}$ candidates [15]. In the RPM, QPO frequencies are assumed to be related to fundamental frequencies of a test particle orbiting a central object. The twin higher frequencies are regarded as the azimuthal frequency $v_{\phi}$ and the periastron precession frequency $v_{\text {per }}$ of quasi-circular orbits in the innermost disk region, respectively. The low-frequency QPO is identified as the nodal precession frequency $v_{\text {nod, }}$ which is emitted at the same radius where the twin higher frequencies are generated.

On the other hand, curvature singularities can be formed during a gravitational collapse. It is commonly believed that singularities can be avoided through quantum gravitational effects. Consequently, since Bardeen proposed the first regular black hole [39], constructing and studying classical black holes without singularities have been a topic of considerable interest in GR and astrophysics communities due to their non-singular property [40-47]. Recently, Simpson and Visser proposed a static and spherically symmetric regular spacetime described by the line element (dubbed the SV metric henceforth),

$$
\begin{aligned}
d s^{2}= & -\left(1-\frac{2 M}{\sqrt{r^{2}+\ell^{2}}}\right) d t^{2}+\left(1-\frac{2 M}{\sqrt{r^{2}+\ell^{2}}}\right)^{-1} d r^{2} \\
& +\left(r^{2}+\ell^{2}\right)\left(d \theta^{2}+\sin ^{2} \theta d \phi^{2}\right)
\end{aligned}
$$

where $M \geq 0$ represents the ADM mass, and $\ell>0$ is a parameter responsible for regularizing the center singularity [48-50]. The most appealing feature of the SV metric (1) is that it can smoothly interpolate between a regular black hole for $\ell<2 M$ and a wormhole for $\ell \geq 2 M$. In the limit of $\ell=0$, the SV metric reduces to the ordinary Schwarzschild spacetime. Subsequently, the properties of the SV metric were discussed, e.g., quasinormal modes [51], precessing and periodic geodesic motions [52], gravitational lensing [53-55] and shadows [56,57]. To make the SV metric more relevant to realistic situations, the SV metric (1) was generalized to a family of rotating black hole mimickers, dubbed the rotating $\mathrm{SV}$ metric, which may represent a rotating wormhole and a rotating regular black hole with one or two horizons [58]. The strong gravitational lensing and the shadows of the rotating SV metric have been investigated in $[59,60]$, respectively.

In this paper, we test gravity with QPOs frequencies observed from GRO J1655-40 within the RPM for the rotat- ing SV metric. The content of this paper is as follows. After we briefly review the RPM and the rotating SV metric in Sects. 2 and 3, respectively, the epicyclic frequencies of the rotating SV are computed in Sect. 3. In Sect. 4, we use the data of GRO J1655-40 to put constraints on the parameters of the rotating SV metric. Section 5 is devoted to our conclusions. Throughout the paper, we use units in which $G=c=1$.

\section{Epicyclic frequencies}

In this section, we consider the timelike geodesic equations in a stationary and axially symmetric spacetime, and then derive the expressions of the epicyclic frequencies. The metric of a stationary and axially symmetric spacetime which satisfies the circularity condition is given by [61]

$d s^{2}=g_{t t} d t^{2}+g_{r r} d r^{2}+2 g_{t \phi} d t d \phi+g_{\theta \theta} d \theta^{2}+g_{\phi \phi} d \phi^{2}$,

where the metric $g_{\mu \nu}$ is a function of $r$ and $\theta$, and we drop the coordinate dependence of the metric functions to simplify the notation. For a massive particle travelling along a time-like world line $x^{\mu}(\tau)$ with $\tau$ being the proper time, the four-velocity $U^{\mu}$ is defined by $U^{\mu}=\dot{x}^{\mu}=d x^{\mu} / d \tau$, which satisfies $U^{\mu} U_{\mu}=-1$. Due to the stationarity and axisymmetry, the metric (2) admits two Killing vectors,

$$
\begin{aligned}
& K^{\mu}=\left(\partial_{t}\right)^{\mu}=(1,0,0,0) \quad \text { and } \\
& R^{\mu}=\left(\partial_{\phi}\right)^{\mu}=(0,0,0,1)
\end{aligned}
$$

The two Killing vectors correspond to two conserved quantities of geodesic motion,

$$
\begin{aligned}
E & =-K_{\mu} \frac{d x^{\mu}}{d \tau}=-g_{t t} \dot{t}-g_{t \phi} \dot{\phi}, \\
L_{z} & =R_{\mu} \frac{d x^{\mu}}{d \tau}=g_{t \phi} \dot{t}+g_{\phi \phi} \dot{\phi},
\end{aligned}
$$

which can be interpreted as the energy per unit mass and the angular momentum per unit mass along the axis of symmetry, respectively. In terms of $E$ and $L_{z}$, one can express $\dot{t}$ and $\dot{\phi}$ as

$\dot{t}=\frac{g_{\phi \phi} E+g_{t \phi} L_{z}}{g_{t \phi}^{2}-g_{t t} g_{\phi \phi}}, \dot{\phi}=\frac{g_{t \phi} E+g_{t t} L_{z}}{g_{t t} g_{\phi \phi}-g_{t \phi}^{2}}$.

Using the above equations, we can rewrite $U^{\mu} U_{\mu}=-1$ as

$g_{r r} \dot{r}^{2}+g_{\theta \theta} \dot{\theta}^{2}=V_{\text {eff }}(r, \theta)$,

where the effective potential $V_{\text {eff }}(r, \theta)$ is defined as

$V_{\mathrm{eff}}(r, \theta)=\frac{E^{2} g_{\phi \phi}+2 E L_{z} g_{t \phi}+L_{z}^{2} g_{t t}}{g_{t \phi}^{2}-g_{t t} g_{\phi \phi}}-1$. 
We consider a circular geodesic at $r=\bar{r}$ on the equatorial plane with $\theta=\pi / 2$, which means that the effective potential may develop a double root at $r=\bar{r}$ on the equatorial plane, i.e., $V_{\text {eff }}(\bar{r}, \pi / 2)=\partial_{r} V_{\text {eff }}(\bar{r}, \pi / 2)=0$. Along the circular orbit, the angular velocity of the particle measured by an observer at infinity is defined by

$\Omega_{\phi}=\frac{d \phi}{d t}=\frac{\dot{\phi}}{\dot{t}}=-\left.\frac{g_{t \phi} E+g_{t t} L_{z}}{g_{\phi \phi} E+g_{t \phi} L_{z}}\right|_{r=\bar{r}, \theta=\pi / 2}$.

Solving $V_{\text {eff }}(\bar{r}, \pi / 2)=0$ with Eq. (8) gives the specific energy and angular momentum of the particle,

$$
\begin{gathered}
E=-\left.\frac{g_{t t}+g_{t \phi} \Omega_{\phi}}{\sqrt{-g_{t t}-2 g_{t \phi} \Omega_{\phi}-g_{\phi \phi} \Omega_{\phi}^{2}}}\right|_{r=\bar{r}, \theta=\pi / 2}, \\
L_{z}=\left.\frac{g_{t \phi}+g_{\phi \phi} \Omega_{\phi}}{\sqrt{-g_{t t}-2 g_{t \phi} \Omega_{\phi}-g_{\phi \phi} \Omega_{\phi}^{2}}}\right|_{r=\bar{r}, \theta=\pi / 2} .
\end{gathered}
$$

Solving $\partial_{r} V_{\text {eff }}(\bar{r}, \pi / 2)=0$ for $\Omega_{\phi}$, one obtains the angular velocity of the particle,

$$
\Omega_{\phi}=\left.\frac{-\partial_{r} g_{t \phi} \pm \sqrt{\left(\partial_{r} g_{t \phi}\right)^{2}-\left(\partial_{r} g_{t t}\right)\left(\partial_{r} g_{\phi \phi}\right)}}{\partial_{r} g_{\phi \phi}}\right|_{r=\bar{r}, \theta=\pi / 2},
$$

where the sign $+/-$ corresponds to a prograde/retrograde orbit. The stability of the circular orbit is determined by the sign of $\partial_{r}^{2} V_{\text {eff }}(\bar{r}, \pi / 2)$, i.e., $\partial_{r}^{2} V_{\text {eff }}(\bar{r}, \pi / 2)>0 \Leftrightarrow$ unstable and $\partial_{r}^{2} V_{\text {eff }}(\bar{r}, \pi / 2)<0 \Leftrightarrow$ stable. The transition between stable and unstable circular orbits, which is determined by $\partial_{r}^{2} V_{\text {eff }}\left(r_{\text {ISCO }}, \pi / 2\right)=0$, is the ISCO, which is located at $r=r_{\text {ISCO }}$ on the equatorial plane.

To derive the epicyclic frequencies associated with the circular orbit, we consider small perturbations of the orbit in both the radial and the vertical directions,

$r(t)=\bar{r}+\delta r(t), \quad \theta(t)=\frac{\pi}{2}+\delta \theta(t)$.

Inserting Eq. (11) into Eq. (6) yields the differential equations for the perturbations $\delta r(t)$ and $\delta \theta(t)$,

$\frac{d^{2} \delta r(t)}{d t^{2}}+\Omega_{r}^{2} \delta r(t)=0, \quad \frac{d^{2} \delta \theta(t)}{d t^{2}}+\Omega_{\theta}^{2} \delta \theta(t)=0$,

where the frequencies of the oscillations are

$$
\Omega_{r}^{2}=-\left.\frac{1}{2 g_{r r} \dot{t}^{2}} \frac{\partial^{2} V_{\text {eff }}(r, \theta)}{\partial r^{2}}\right|_{r=\bar{r}, \theta=\pi / 2}, \Omega_{\theta}^{2}
$$

$$
=-\left.\frac{1}{2 g_{\theta \theta} \dot{t}^{2}} \frac{\partial^{2} V_{\mathrm{eff}}(r, \theta)}{\partial \theta^{2}}\right|_{r=\bar{r}, \theta=\pi / 2} .
$$

We then define $v_{\phi}=\Omega_{\phi} / 2 \pi, v_{r}=\Omega_{r} / 2 \pi$ and $v_{\theta}=\Omega_{\theta} / 2 \pi$ as the azimuthal, radial and vertical epicyclic frequencies, respectively. The periastron precession frequency $v_{\text {per }}$ and the nodal precession frequency $v_{\text {nod }}$ are defined by $v_{\text {per }}=$ $v_{\phi}-v_{r}$ and $v_{\text {nod }}=v_{\phi}-v_{\theta}$, respectively.

\section{Rotating Simpson-Visser metric}

In [58], a rotating generalization of the static and spherically symmetric metric (1) has been constructed by employing the Newman-Janis procedure [62]. This stationary and axially symmetric metric can describe a rotating traversable wormhole and a rotating regular black hole with one or two horizons. In particular, the rotating SV metric reads

$$
\begin{aligned}
d s^{2}= & -\left(1-\frac{2 M \sqrt{r^{2}+\ell^{2}}}{\Sigma}\right) d t^{2} \\
& +\frac{\Sigma}{\Delta} d r^{2}+\Sigma d \theta^{2}-\frac{4 M a \sin ^{2} \theta \sqrt{r^{2}+\ell^{2}}}{\Sigma} d t d \phi \\
& +\frac{A \sin ^{2} \theta}{\Sigma} d \phi^{2},
\end{aligned}
$$

with

$\Sigma=r^{2}+\ell^{2}+a^{2} \cos ^{2} \theta$,

$\Delta=r^{2}+\ell^{2}+a^{2}-2 M \sqrt{r^{2}+\ell^{2}}$,

$A=\left(r^{2}+\ell^{2}+a^{2}\right)^{2}-\Delta a^{2} \sin ^{2} \theta$,

where $a$ is the spin parameter. The rotating SV metric will reduce to the SV metric (1) if $a=0$ and to the Kerr metric if $\ell=0$. Interestingly, the rotating SV metric is everywhere regular when $\ell>0$ [58].

The horizons of the rotating SV metric are determined by $\Delta=0$, whose solutions are

$r_{ \pm}=\sqrt{\left(M \pm \sqrt{M^{2}-a^{2}}\right)^{2}-\ell^{2}}$.

As shown in [58], the phases of the rotating SV metric are determined by the existence of $r_{ \pm}$. Specifically, the rotating SV metric represents

- a traversable wormhole: $M<a$ or $\ell>M+\sqrt{M^{2}-a^{2}}$;

- a regular black hole with one horizon (RBH-I): $M-$ $\sqrt{M^{2}-a^{2}}<\ell<M+\sqrt{M^{2}-a^{2}}$ and $M>a$;

- a regular black hole with two horizons (RBH-II): $\ell<$ $M-\sqrt{M^{2}-a^{2}}$ and $M>a$;

- there limiting cases: a one-way wormhole with a null throat when $\ell=M+\sqrt{M^{2}-a^{2}}$, a regular black 

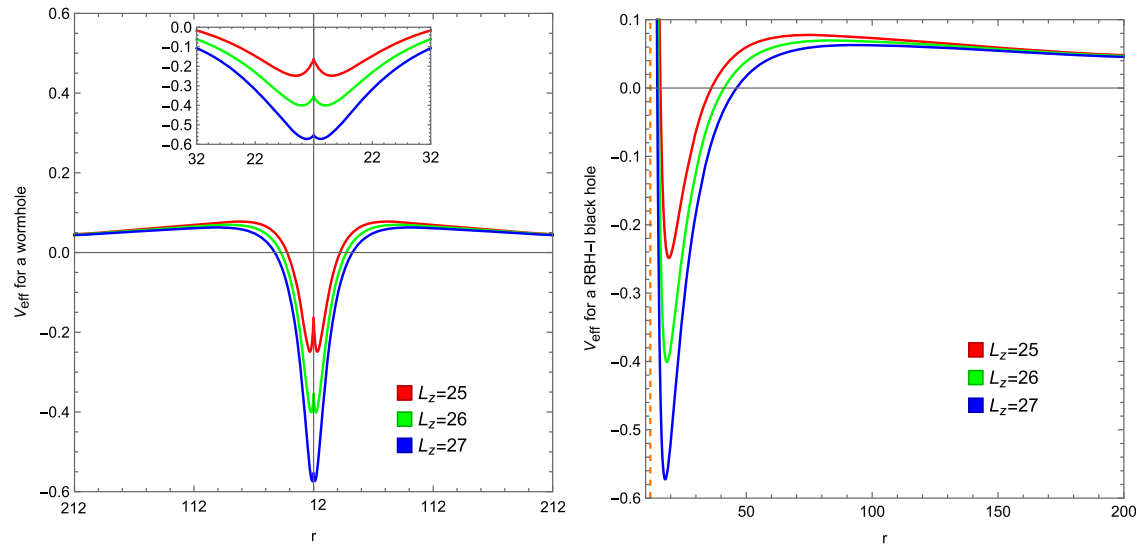

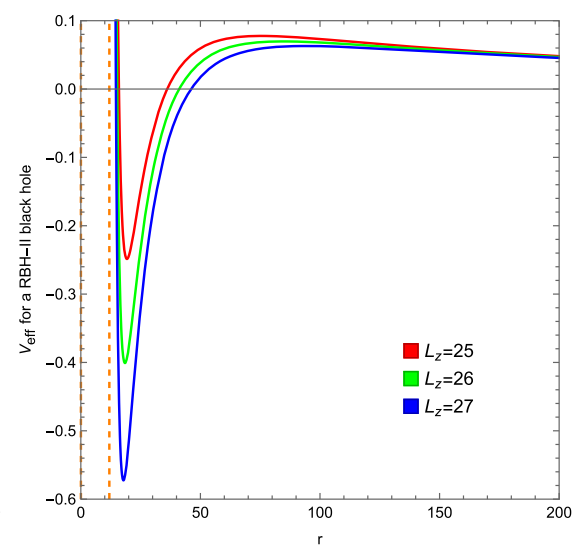

Fig. 1 Plots of the effective potential $V_{\text {eff }}$ of a massive particle travelling on the equatorial plane with $\theta=\pi / 2$ as a function of radius $r$ in a traversable wormhole with $\ell=12$ (left panel), a RBH-I black hole with $\ell=1$ (middle panel) and a RBH-II black hole with $\ell=0.08$ (right panel) for $L_{z}=25$ (red lines), $L_{z}=26$ (green lines) and $L_{z}=27$ (blue lines). We have $M=6, a=1$ and $E=1$. The horizons of the RBH-I and RBH-II black holes are represented by orange dashed lines.

hole with one horizon and a null throat when $\ell=$ $M-\sqrt{M^{2}-a^{2}}$ and an extremal regular black hole when $M=a$.

Substituting the rotating SV metric (14) into Eq. (7), one can obtain the effective potential $V_{\text {eff }}$ for a massive particle in the rotating SV metric. Like a Schwarzschild black, we find that, for a massive particle with a large enough $\left|\mathbf{L}_{\mathbf{z}}\right|$, $V_{\text {eff }}$ possesses a minimum and a maximum on the equatorial plane, corresponding to a stable circular orbit and an unstable one, outside the event horizon of a black hole or in the each universe of a wormhole. As $\left|\mathbf{L}_{\mathbf{z}}\right|$ increases, the two circular orbits move further away from each other. In Fig. 1, we plot $V_{\text {eff }}$ as a function of $r$ for various values of $L_{z}$ in three cases, i.e., a traversable wormhole, a RBH-I black hole and a RBHII black hole. Here without loss of generality, we have set $M=6, E=1, a=1$ and $\theta=\pi / 2$. Note that the two universes of the wormhole are connected at $r=\ell$, where the effective potential has a cusp.

For a circular orbit at $r=\bar{r}$ on the equatorial plane, substituting the rotating SV metric (14) into Eqs. (8) and (13) gives epicyclic frequencies in the rotating $\mathrm{SV}$ metric,

$$
\begin{aligned}
v_{\phi} & =\frac{1}{2 \pi} \frac{M^{1 / 2}}{\left(\bar{r}^{2}+\ell^{2}\right)^{3 / 4} \pm a M^{1 / 2}}, \\
v_{r} & =\frac{\bar{r} v_{\phi}}{\left(\bar{r}^{2}+\ell^{2}\right)^{1 / 2}} \\
& \sqrt{1-\frac{6 M}{\left(\bar{r}^{2}+\ell^{2}\right)^{1 / 2}}-\frac{3 a^{2}}{\left(\bar{r}^{2}+\ell^{2}\right)} \pm \frac{8 a M^{1 / 2}}{\left(\bar{r}^{2}+\ell^{2}\right)^{3 / 4}}}, \\
v_{\theta}= & v_{\phi} \sqrt{1 \mp \frac{4 a M^{1 / 2}}{\left(\bar{r}^{2}+\ell^{2}\right)^{3 / 4}}+\frac{3 a^{2}}{\left(\bar{r}^{2}+\ell^{2}\right)}},
\end{aligned}
$$

In the three cases, the effective potential always decreases to zero at infinity. For the traversable wormhole, the effective potential has one maximum and one minimum in each universe. For the RBH-I black hole, the effective potential has one maximum and one minimum outside the event horizon. The effective potential of the RBH-II black hole is similar to that of the RBH-I black hole

which can be used to constrain the four parameters $\ell, \bar{r}$, $M$, and $a$ of the rotating SV metric. Here, the top/bottom row of the \pm and $\mp$ signs corresponds to the orbit corotating/counter-rotating with the spacetime. To illustrate the dependence of the epicyclic frequencies on $\ell$, we plot $v_{\phi}$, $v_{\text {per }}$ and $v_{\text {nod }}$ as a function of $\ell$ for various values of $a$ in Fig. 2, where $M=1$ and $\bar{r}=6$. The prograde and retrograde cases are represented by solid and dashed lines, respectively. The left panel shows that, for a fixed $a$, the azimuthal epicyclic frequency $v_{\phi}$ decreases with $\ell$ increasing in both prograde and retrograde cases, whereas $v_{\phi}$ of the prograde orbit is smaller than that of the retrograde orbit. The periastron precession frequency $v_{\text {per }}$ is displayed in the middle panel, and also decreases as $\ell$ increases for both prograde and retrograde orbits. Like $v_{\phi}$, the retrograde orbits have larger $v_{\text {per. }}$. Note that retrograde circular orbits of radius $\bar{r}=6$ do not exist when $\ell$ is small enough. However, as shown in the right panel, the nodal precession frequency $v_{\text {nod }}$ of the prograde/retrograde orbits decreases/increases as $\ell$ increases for a given $a$. More interestingly, when $a>0$, $v_{\text {nod }}$ of the retrograde orbits is negative while that of the prograde orbits is positive. Note that the nodal precession is defined as the precession of the ascending node, which is the point where the orbit passes through the plane of reference, e.g., the equatorial plane in our paper. If the SV metric is spherically symmetric with $a=0$, the orbital plane of a particle remains fixed in space, and hence the nodal precession $v_{\text {nod }}$ vanishes, which can be seen from Fig. 2. However for the rotating SV metric with a nonzero $a$, a particle in a prograde/retrograde orbit has been shown to have a positive/negative nodal precession $v_{\text {nod, }}$, which means that the orbital plane rotates in the same/opposite direction as the direction of the SV metric's 

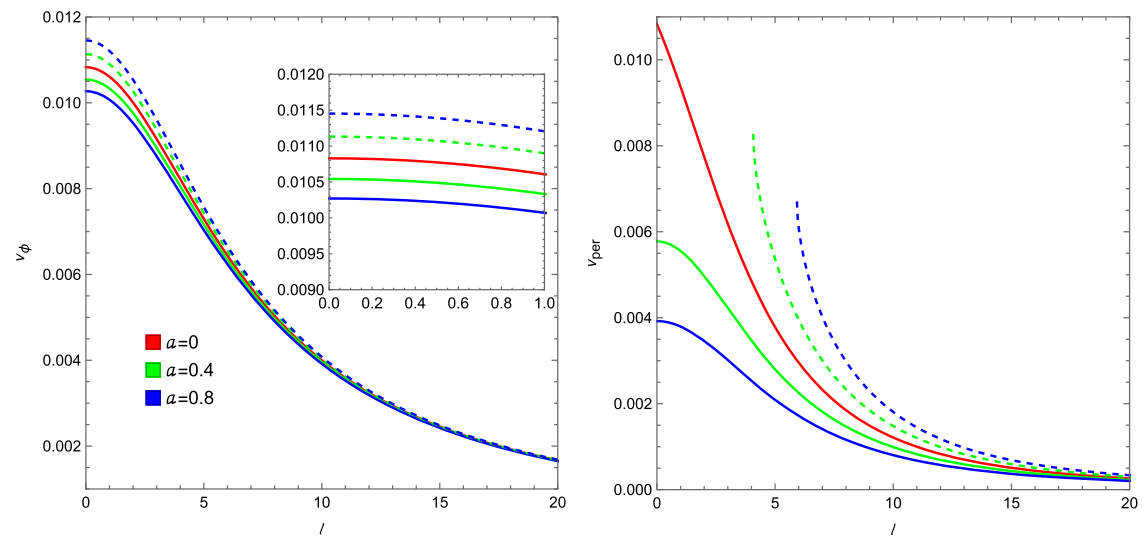

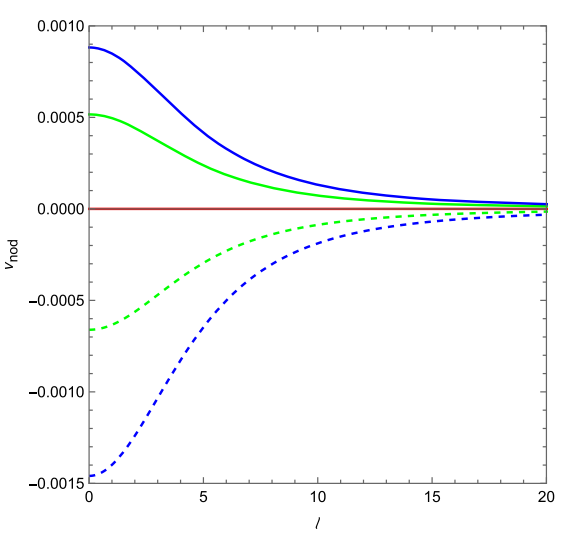

Fig. 2 Plots of the azimuthal epicyclic frequency $v_{\phi}$ (left panel), the periastron precession frequency $v_{\text {per }}$ (middle panel) and the nodal precession frequency $v_{\text {nod }}$ (right panel) of prograde (solid lines) and retrograde (dashed lines) orbits at $\bar{r}=6$ as a function of $\ell$ for $a=0$ (red lines), $a=0.4$ (green lines) and $a=0.8$ (blue lines) in the rotating
SV metric with $M=1$. The $a=0$ case corresponds to the SV metric, which has $v_{\text {nod }}=0$ due to spherical symmetry. Except $v_{\text {nod }}$ with $a=0$, the magnitudes of $v_{\phi}, v_{\text {per }}$ and $v_{\text {nod }}$ decrease as $\ell$ grows with a fixed $a$. For the retrograde orbits in the rotating SV metric with $a>0$, the values of $v_{\text {nod }}$ are shown to be negative rotation. Interestingly, it is well-known that the nodal precessions of a satellite in prograde and retrograde orbits around Earth have opposite signs [63]. Finally, it is noteworthy that the ISCO radius $r_{\text {ISCO }}$ is determined by [58],

$r_{\mathrm{ISCO}}^{2}+\ell^{2}-6 M \sqrt{r_{\mathrm{ISCO}}^{2}+\ell^{2}} \pm 8 a \sqrt{M \sqrt{r_{\mathrm{ISCO}}^{2}+\ell^{2}}}=3 a^{2}$,

where $+/-$ is associated with the prograde/retrograde ISCO.

\section{Constraining rotating Simpson-Visser metric by quasi-periodic oscillations}

In this section, we use the RPM along with the QPO frequencies from GRO J1655-40 to put constraints on the parameters of the rotating SV metric. GRO J1655-40 is an X-ray binary, consisting of a primary star and a compact companion [64]. The measurement of the X-ray spectrum was found to exhibit type-C low-frequency QPOs and simultaneous highfrequency QPOs, which are observed in pairs and therefore dubbed lower and upper high-frequency QPOs $[12,14]$. In particular, we consider two sets of QPOs with the observed frequencies based on the RXTE observations [14],

$$
\begin{array}{cl}
v_{1 \mathrm{U}}=441 \mathrm{~Hz}, & \sigma_{1 \mathrm{U}}=2 \mathrm{~Hz}, \\
v_{1 \mathrm{~L}}=298 \mathrm{~Hz}, & \sigma_{1 \mathrm{~L}}=4 \mathrm{~Hz}, \\
v_{1 \mathrm{C}}=17.3 \mathrm{~Hz}, & \sigma_{1 \mathrm{C}}=0.1 \mathrm{~Hz}
\end{array}
$$

and

$$
v_{2 \mathrm{U}}=451 \mathrm{~Hz}, \quad \sigma_{2 \mathrm{U}}=5 \mathrm{~Hz},
$$

$$
\begin{gathered}
\nu_{2 \mathrm{~L}}=- \\
\nu_{2 \mathrm{C}}=18.3 \mathrm{~Hz}, \quad \sigma_{2 \mathrm{C}}=0.1 \mathrm{~Hz} .
\end{gathered}
$$

In the RPM, three simultaneous QPO frequencies are generated at the same radial coordinate in the accretion disk. The upper high-frequency QPOs correspond to the azimuthal epicyclic frequency $v_{\phi}$, the lower high-frequency QPOs to the periastron precession frequency $v_{\text {per }}$, and the low-frequency QPOs to the nodal precession frequency $v_{\text {nod }}$. Moreover, it is reasonable to assume that the above two sets of QPOs result from two circular orbits of different radii, i.e., $\bar{r}_{1}$ and $\bar{r}_{2}$. In short, we have five free parameters: the mass $M$, the spin parameter $a$, the $\ell$ parameter, and the radii $\bar{r}_{1}$ and $\bar{r}_{2}$ corresponding to the QPOs with three frequencies and two frequencies, respectively. To obtain the estimate of the five parameters from the observed QPO frequencies, we follow the procedure used in $[28,33,34]$ and perform a $\chi$-square analysis with

$$
\begin{aligned}
\chi^{2}\left(M, a, \ell, \bar{r}_{1}, \bar{r}_{2}\right)= & \frac{\left(v_{1 \phi}-v_{1 \mathrm{U}}\right)^{2}}{\sigma_{1 \mathrm{U}}}+\frac{\left(v_{1 \mathrm{per}}-v_{1 \mathrm{~L}}\right)^{2}}{\sigma_{1 \mathrm{~L}}} \\
& +\frac{\left(v_{1 \mathrm{nod}}-v_{1 \mathrm{C}}\right)^{2}}{\sigma_{1 \mathrm{C}}} \\
& +\frac{\left(v_{2 \phi}-v_{2 \mathrm{U}}\right)^{2}}{\sigma_{2 \mathrm{U}}}+\frac{\left(\nu_{2 \mathrm{nod}}-v_{2 \mathrm{C}}\right)^{2}}{\sigma_{2 \mathrm{C}}},
\end{aligned}
$$

the minimum of which, $\chi_{\min }^{2}$, occurs at the best estimate of $M, a \ell, \bar{r}_{1}$ and $\bar{r}_{2}$. The range of the parameters at a confidence level (C.L.) is determined by the interval $\chi_{\min }^{2}+\Delta \chi^{2}$. In the case of five degrees of freedom, the intervals with $\Delta \chi^{2}=5.89,11.29$ and 17.96 correspond to $68.3 \%, 95.4 \%$ and $99.7 \%$ C.L., respectively, which are the probability inter- 

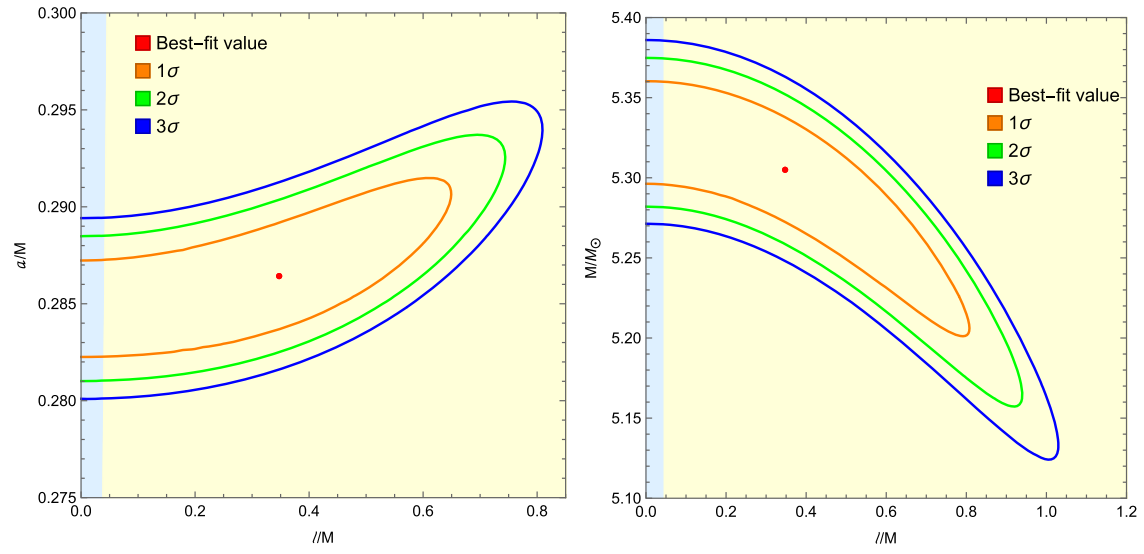

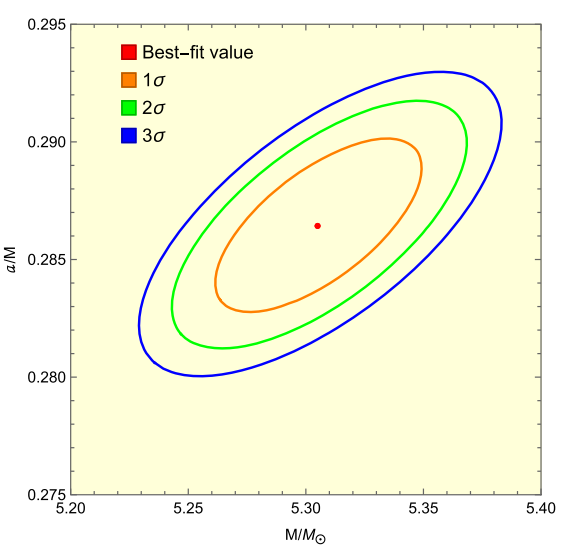

Fig. 3 Estimate of the mass $M / M_{\odot}$, of the spin parameter $a / M$ and of the parameter $\ell / M$ of the rotating SV metric, which describes the spacetime around the compact object in GRO J1655-40, from interpreting the observations of QPOs in the RPM. Specifically, the best-fit values (red dots) and the contour levels of $1 \sigma$ (orange lines), $2 \sigma$ (green lines) and $3 \sigma$ (blue lines) for the parameters are shown in the $\ell / M-a / M$ (left panel), $\ell / M-M / M_{\odot}$ (middle panel) and $M / M_{\odot}-a / M$ (right panel) planes. In the yellow and blue regions, the rotating SV metric represents regu- lar black hole solutions with one and two horizons, respectively. The observed frequencies of the QPOs in GRO J1655-40 are consistent with a Kerr black hole (the rotating SV metric with $\ell=0$ ), but they also allow for large deviations from the Kerr black hole solution. In particular, a regular black hole with one horizon is favored over the other phases of the rotating SV metric, e.g., a regular black hole with two horizons and a traversable wormhole vals designated as 1, 2, and 3 standard deviation limits, respectively.

Computing $\chi^{2}$, we find $\chi_{\min }^{2}=0.195$ and obtain the best fits of the parameters of the rotating SV metric within $68.3 \%$ credibility,

$$
\begin{aligned}
M / M_{\odot} & =5.305_{-0.028}^{+0.041}, a / M=0.286_{-0.002}^{+0.003}, \ell / \\
M & =0.347_{-0.347}^{+0.011}
\end{aligned}
$$

Note that our result is consistent with the measurement of the mass $M$ by optical and infrared observations, which give $M=5.4 \pm 0.3 M_{\odot}[23]$. We present the best estimate and the $1 \sigma, 2 \sigma$ and $3 \sigma$ contour levels of $M / M_{\odot}, a / M$ and $\ell / M$ in Fig. 3, where the yellow/blue regions represent the RBH$\mathrm{I} / \mathrm{RBH}-\mathrm{II}$ phases of the rotating SV metric. As shown in the left and middle panels, while the hypothesis that the compact object of GRO J1655-40 is described by a Kerr black hole is consistent with the interpretation of the QPOs' data in the RPM, significant deviations from the Kerr metric are allowed. In fact, the best-fit values are in the parametric region of the RBH-I phase, and the regions within 1-, 2- and 3-standard deviation limits are almost in the RBH-I region. Therefore, the observation of GRO J1655-40 favors a regular black hole with one horizon if the compact object of GRO J1655-40 is described by the rotating SV metric. The best-fit values of the radii of the circular orbits associated with the two sets of QPOs are found to be $\bar{r}_{1}=5.669 M=1.1304 r_{\text {ISCO }}$ and $\bar{r}_{2}=5.563 \mathrm{M}=1.1094 r_{\mathrm{ISCO}}$, respectively, where $r_{\text {ISCO }}=5.105 M$ is the innermost stable circular orbit evaluated for the rotating SV metric with the best-fit values (22). Consequently, the two circular orbits responsible for gener- ating the two sets of QPOs lie in the close vicinity of the ISCO, and hence are in the strong-field region of the rotating SV metric.

\section{Conclusions}

In this paper, we explored potential deviations from the GR predictions of astrophysical black holes using QPOs observed in the power density spectrum of GRO J1655-40. Specially, we modelled the spacetime around the compact object of GRO J1655-40 by the rotating SV metric, and interpreted the observed QPOs within the RPM, which relates the QPO frequencies to epicyclic frequencies of geodesics. The rotating SV metric reduces to a Kerr black hole in the limit of $\ell=0$, and possesses multiple phases, e.g., a regular black hole with one or two horizons and a traversable wormhole. To test the nature of the the compact object of GRO J1655-40, we performed a $\chi^{2}$ analysis by fitting the QPO frequencies computed in the RPM with the observations of two sets of QPOs from GRO J1655-40. Our results show a preference towards a regular black hole with one horizon compared to the Kerr black hole predicted in GR.

Acknowledgements We thank Guangzhou Guo for his helpful discussions and suggestions. Houwen $\mathrm{Wu}$ is supported by the International Visiting Program for Excellent Young Scholars of Sichuan University. This work is supported in part by NSFC (Grant No. 11875196, 11375121, 11947225 and 11005016).

Data Availability Statement This manuscript has no associated data or the data will not be deposited. [Authors' comment: Data sharing 
not applicable to our article as no datasets were generated or analyzed during the current study.]

Open Access This article is licensed under a Creative Commons Attribution 4.0 International License, which permits use, sharing, adaptation, distribution and reproduction in any medium or format, as long as you give appropriate credit to the original author(s) and the source, provide a link to the Creative Commons licence, and indicate if changes were made. The images or other third party material in this article are included in the article's Creative Commons licence, unless indicated otherwise in a credit line to the material. If material is not included in the article's Creative Commons licence and your intended use is not permitted by statutory regulation or exceeds the permitted use, you will need to obtain permission directly from the copyright holder. To view a copy of this licence, visit http://creativecomm ons.org/licenses/by/4.0/.

Funded by SCOAP ${ }^{3}$.

\section{References}

1. B.P. Abbott et al., Observation of gravitational waves from a binary black hole merger. Phys. Rev. Lett. 116(6), 061102 (2016). https:// doi.org/10.1103/PhysRevLett.116.061102. arXiv:1602.03837

2. K. Akiyama et al., First M87 event horizon telescope results. I. The shadow of the supermassive black hole. Astrophys. J. Lett 875, L1 (2019). https://doi.org/10.3847/2041-8213/ab0ec7. arXiv: 1906.11238

3. M. van der Klis, Millisecond oscillations in x-ray binaries. Ann. Rev. Astron. Astrophys. 38, 717-760 (2000). https://doi.org/10. 1146/annurev.astro.38.1.717. arXiv:astro-ph/0001167

4. E. Berti et al., Testing general relativity with present and future astrophysical observations. Class. Quantum Gravity 32, 243001 (2015). https://doi.org/10.1088/0264-9381/32/24/ 243001. arXiv: 1501.07274

5. D. Psaltis, Probes and tests of strong-field gravity with observations in the electromagnetic spectrum. Living Rev. Relativ. 11, 9 (2008). https://doi.org/10.12942/lrr-2008-9. arXiv:0806.1531

6. D. Psaltis, Two approaches to testing general relativity in the strongfield regime. J. Phys: Conf. Ser. 189, 012033 (2009). https://doi. org/10.1088/1742-6596/189/1/012033. arXiv:0907.2746

7. K. Jusufi, M. Azreg-Aïnou, M. Jamil, S.-W. Wei, W. Qiang, A. Wang, Quasinormal modes, quasiperiodic oscillations, and the shadow of rotating regular black holes in nonminimally coupled Einstein-Yang-Mills theory. Phys. Rev. D 103(2), 024103 (2021). https://doi.org/10.1103/PhysRevD.103.024013. arXiv:2008.08450

8. M. Azreg-Aïnou, Z. Chen, B. Deng, M. Jamil, T. Zhu, W. Qiang, Y.-K. Lim, Orbital mechanics and quasiperiodic oscillation resonances of black holes in Einstein-Æther theory. Phys. Rev. D 102(4), 044028 (2020). https://doi.org/10.1103/PhysRevD.102. 044028. arXiv:2004.02602

9. A. Ingram, S. Motta, A review of quasi-periodic oscillations from black hole X-ray binaries: observation and theory. New Astron. Rev. 85, 101524 (2019). https://doi.org/10.1016/j.newar. 2020.101524. arXiv:2001.08758

10. E.H. Morgan, R.A. Remillard, J. Greiner, RXTE observations of QPOs in the black hole candidate GRS 1915+105. Astrophys. J. 482, 993-1010 (1997). https://doi.org/10.1086/304191

11. R.A. Remillard, E.H. Morgan, J.E. McClintock, C.D. Bailyn, J.A. Orosz, Rxte observations of $0.1-300 \mathrm{~Hz}$ quasi-periodic oscillations in the microquasar gro j1655-40. Astrophys. J. 522(1), 397 (1999)

12. T.E. Strohmayer, Discovery of a $450 \mathrm{hz}$ quasi-periodic oscillation from the microquasar gro $\mathrm{j} 1655-40$ with the rossi $\mathrm{x}$-ray timing explorer. Astrophys. J. Lett. 522(1), L49 (2001)
13. M.A. Abramowicz, W. Kluzniak, A Precise determination of angular momentum in the black hole candidate GRO J1655-40. Astron. Astrophys. 374, L19 (2001). https://doi.org/10.1051/0004-6361: 20010791. arXiv:astro-ph/0105077

14. S.E. Motta, T.M. Belloni, L. Stella, T. Muñoz Darias, R. Fender, Precise mass and spin measurements for a stellar-mass black hole through X-ray timing: the case of GRO J1655-40. Mon. Not. R. Astron. Soc 437(3), 2554-2565 (2014). https://doi.org/10.1093/ mnras/stt2068. arXiv:1309.3652

15. L. Stella, M. Vietri, S. Morsink, Correlations in the qpo frequencies of low mass x-ray binaries and the relativistic precession model. Astrophys. J. Lett. 524, L63-L66 (1999). https://doi.org/10.1086/ 312291. arXiv:astro-ph/9907346

16. A. Cadez, M. Calvani, U. Kostic, On the tidal evolution of the orbits of low-mass satellites around black holes. Astron. Astrophys. 487, 527-532 (2008). https://doi.org/10.1051/0004-6361: 200809483. arXiv:0809.1783

17. P. Rebusco, Twin peaks kHz QPOs: mathematics of the $3: 2$ orbital resonance. Publ. Astron. Soc. Jap. 56, 553 (2004). https://doi.org/ 10.1093/pasj/56.3.553. arXiv:astro-ph/0403341

18. W. Kluzniak, M.A. Abramowicz. Parametric epicyclic resonance in black hole disks: qpos in micro-quasars (2002). arXiv:astro-ph/0203314

19. M.A. Abramowicz, W. Kluzniak, Z. Stuchlik, G. Torok, The orbital resonance model for twin peak $\mathrm{kHz}$ QPOs: measuring the black hole spins in microquasars (2004). arXiv:astro-ph/0401464

20. S. Kato, Frequency correlations of QPOs based on a disk oscillation model in warped disks. Publ. Astron. Soc. Jap. 59, 451 (2007). https://doi.org/10.1093/pasj/59.2.451. arXiv:astro-ph/0701085

21. M. Bursa, M.A. Abramowicz, V. Karas, W. Kluzniak, The upper kHz QPO: a gravitationally lensed vertical oscillation. Astrophys. J. Lett 617, L45-L48 (2004). https://doi.org/10.1086/427167

22. G. Torok, P. Bakala, E. Sramkova, Z. Stuchlik, M. Urbanec, On mass-constraints implied by the relativistic precession model of twin-peak quasi-periodic oscillations in Circinus X-1. Astrophys. J. 714, 748-757 (2010). https://doi.org/10.1088/0004-637X/714/ 1/748. arXiv: 1008.0088

23. M.E. Beer, P. Podsiadlowski, The quiescent light curve and evolutionary state of gro j1655-40. Mon. Not. R. Astron. Soc. 331, 351 (2002). https://doi.org/10.1046/j.1365-8711.2002. 05189.x. arXiv:astro-ph/0109136

24. T. Johannsen, D. Psaltis, Testing the no-hair theorem with observations in the electromagnetic spectrum. III. Quasi-periodic variability. Astrophys. J. 726, 11 (2011). https://doi.org/10.1088/ 0004-637X/726/1/11. arXiv: 1010.1000

25. A.K. Kulkarni, R.F. Penna, R.V. Shcherbakov, J.F. Steiner, R. Narayan, A. Sadowski, Y. Zhu, J.E. McClintock, S.W. Davis, J.C. McKinney, Measuring black hole spin by the continuum-fitting method: effect of deviations from the Novikov-Thorne disc model. Mon. Not. R. Astron. Soc. 414, 1183 (2011). https://doi.org/10. 1111/j.1365-2966.2011.18446.x. arXiv:1102.0010

26. G. Pappas, What can quasi-periodic oscillations tell us about the structure of the corresponding compact objects? Mon. Not. R. Astron. Soc. 422, 2581-2589 (2012). https://doi.org/10.1111/j. 1365-2966.2012.20817.x. arXiv:1201.6071

27. C. Bambi, Probing the space-time geometry around black hole candidates with the resonance models for high-frequency QPOs and comparison with the continuum-fitting method. JCAP 09, 014 (2012). https://doi.org/10.1088/1475-7516/2012/09/014. arXiv: 1205.6348

28. C. Bambi, Testing the nature of the black hole candidate in GRO J1655-40 with the relativistic precession model. Eur. Phys. J. C 75(4), 162 (2015). https://doi.org/10.1140/epjc/ s10052-015-3396-7. arXiv:1312.2228

29. A. Maselli, L. Gualtieri, P. Pani, L. Stella, V. Ferrari, Testing gravity with quasi periodic oscillations from accreting black holes: 
the case of the Einstein-Dilaton-Gauss-Bonnet theory. Astrophys. J. 801(2), 115 (2015). https://doi.org/10.1088/0004-637X/801/2/ 115. arXiv: 1412.3473

30. A.G. Suvorov, A. Melatos, Testing modified gravity and no-hair relations for the Kerr-Newman metric through quasiperiodic oscillations of galactic microquasars. Phys. Rev. D 93, 024004 (2016). https://doi.org/10.1103/PhysRevD.93.024004. arXiv:1512.02291

31. C. Bambi, S. Nampalliwar, Quasi-periodic oscillations as a tool for testing the Kerr metric: a comparison with gravitational waves and iron line. EPL 116(3), 30006 (2016). https://doi.org/10.1209/ 0295-5075/116/30006. arXiv: 1604.02643

32. S. Chen, M. Wang, J. Jing, Testing gravity of a regular and slowly rotating phantom black hole by quasi-periodic oscillations. Class. Quantum Gravity 33(19), 195002 (2016). https://doi.org/10.1088/ 0264-9381/33/19/195002. arXiv: 1604.07106

33. A. Allahyari, L. Shao, Testing no-hair theorem by quasiperiodic oscillations: the quadrupole of GRO J1655-40 (2021). arXiv:2102.02232

34. S. Chen, Z. Wang, J. Jing, Testing gravity of a disformal Kerr black hole in quadratic degenerate higher-order scalar-tensor theories by quasi-periodic oscillations. JCAP 06, 043 (2021). https://doi.org/ 10.1088/1475-7516/2021/06/043. arXiv:2103.11788

35. I. Banerjee, S. Chakraborty, S. SenGupta, Looking for extra dimensions in the observed quasi-periodic oscillations of black holes (2021). arXiv:2105.06636

36. V. De Falco, M. De Laurentis, S. Capozziello, Epicyclic frequencies in static and spherically symmetric wormhole geometries (2021). arXiv:2106.12564

37. K. Rink, I. Caiazzo, J. Heyl, Testing general relativity using quasiperiodic oscillations from X-ray black holes: XTE J1550-564 and GRO J1655-40 (2021). arXiv:2107.06828

38. L. Stella, M. Vietri, Lense-Thirring precession and QPOS in low mass X-ray binaries. Astrophys. J. Lett. 492, L59 (1998). https:// doi.org/10.1086/311075. arXiv:astro-ph/9709085

39. J.M. Bardeen, Non-Singular General-Relativistic Gravitational Collapse, in Proceedings of the International Conference GR5, Tbilisi, USSR (1968), p 174

40. T.A. Roman, P.G. Bergmann, Stellar collapse without singularities? Phys. Rev. D 28, 1265-1277 (1983). https://doi.org/10.1103/ PhysRevD.28.1265

41. S.A. Hayward, Formation and evaporation of regular black holes. Phys. Rev. Lett. 96, 031103 (2006). https://doi.org/10.1103/ PhysRevLett.96.031103. arXiv:gr-qc/0506126

42. J.M. Bardeen, Black Hole Evaporation without an Event Horizon, vol. 6 (2014). arXiv:1406.4098

43. V.P. Frolov, Notes on nonsingular models of black holes. Phys. Rev. D 94(10), 104056 (2016). https://doi.org/10.1103/PhysRevD.94. 104056. arXiv: 1609.01758

44. P.A. Cano, S. Chimento, T. Ortín, A. Ruipérez, Regular stringy black holes? Phys. Rev. D 99(4), 046014 (2019). https://doi.org/ 10.1103/PhysRevD.99.046014. arXiv: 1806.08377

45. J.M. Bardeen, Models for the nonsingular transition of an evaporating black hole into a white hole (2018). arXiv:1811.06683

46. R. Carballo-Rubio, F. Di Filippo, S. Liberati, C. Pacilio, M. Visser, On the viability of regular black holes. JHEP 07, 023 (2018). https:// doi.org/10.1007/JHEP07(2018)023. arXiv:1805.02675

47. R. Carballo-Rubio, F. Di Filippo, S. Liberati, M. Visser, Phenomenological aspects of black holes beyond general relativity. Phys. Rev. D 98(12), 124009 (2018). https://doi.org/10.1103/ PhysRevD.98.124009. arXiv:1809.08238
48. A. Simpson, M. Visser, Black-bounce to traversable wormhole. JCAP 02, 042 (2019). https://doi.org/10.1088/1475-7516/2019/ 02/042. arXiv: 1812.07114

49. A. Simpson, P. Martin-Moruno, M. Visser, Vaidya spacetimes, black-bounces, and traversable wormholes. Class. Quantum Gravity 36(14), 145007 (2019). https://doi.org/10.1088/1361-6382/ ab28a5. arXiv: 1902.04232

50. A. Simpson, Traversable wormholes, regular black holes, and black-bounces. Master's thesis, Victoria U., Wellington (2019). arXiv:2104.14055

51. M.S. Churilova, Z. Stuchlik, Ringing of the regular blackhole/wormhole transition. Class. Quantum Gravity 37(7), $07514 \quad$ (2020). https://doi.org/10.1088/1361-6382/ab7717. arXiv: 1911.11823

52. T.-Y.Zhou, Y.Xie, Precessing and periodic motions around a blackbounce/traversable wormhole. Eur. Phys. J. C 80(11), 1070 (2020). https://doi.org/10.1140/epjc/s10052-020-08661-w

53. J.R. Nascimento, A.Y. Petrov, P.J. Porfirio, A.R. Soares, Gravitational lensing in black-bounce spacetimes. Phys. Rev. D 102(4), 044021 (2020). https://doi.org/10.1103/PhysRevD.102.044021. arXiv:2005.13096

54. X.-T. Cheng, Y. Xie, Probing a black-bounce, traversable wormhole with weak deflection gravitational lensing. Phys. Rev. D 103(6), 064040 (2021). https://doi.org/10.1103/PhysRevD.103.064040

55. N. Tsukamoto, Gravitational lensing by two photon spheres in a black-bounce spacetime in strong deflection limits (2021). arXiv:2105.14336

56. K.A. Bronnikov, R.A. Konoplya, T.D. Pappas, General parametrization of wormhole spacetimes and its application to shadows and quasinormal modes. Phys. Rev. D 103(12), 124062 (2021). https://doi.org/10.1103/PhysRevD.103.124062. arXiv:2102.10679

57. M. Guerrero, G.J. Olmo, D. Rubiera-Garcia, D. Sáez-Chillón Gómez, Shadows and optical appearance of black bounces illuminated by a thin accretion disk (2021). arXiv:2105.15073

58. J. Mazza, E. Franzin, S. Liberati, A novel family of rotating black hole mimickers. JCAP 04, 082 (2021). https://doi.org/10.1088/ 1475-7516/2021/04/082. arXiv:2102.01105

59. S. Ul Islam, J. Kumar, S.G. Ghosh, Strong gravitational lensing by rotating Simpson-Visser black holes (2021). arXiv:2104.00696

60. R. Shaikh, K. Pal, K. Pal, T. Sarkar, Constraining alternatives to the Kerr black hole (2021). https://doi.org/10.1093/mnras/stab1779. arXiv:2102.04299

61. S. Chandrasekhar, The mathematical theory of black holes (1985)

62. E.T. Newman, A.I. Janis, Note on the Kerr spinning particle metric. J. Math. Phys. 6, 915-917 (1965). https://doi.org/10.1063/1. 1704350

63. C.D. Brown, Elements of spacecraft design. Aiaa (2002)

64. J.A. Orosz, C.D. Bailyn, Optical observations of GRO J165540 in quiescence I: a precise mass for the black hole primary. Astrophys. J. 477, 876 (1997). https://doi.org/10.1086/303741. arXiv:astro-ph/9610211 